\title{
Efficient conceptual design for LED- based pixel light vehicle headlamps
}

\section{Marcel Philipp Held, Roland Lachmayer}

Marcel Philipp Held, Roland Lachmayer, "Efficient conceptual design for LED-based pixel light vehicle headlamps," Proc. SPIE 10603, Photonics, Devices, and Systems VII, 1060315 (1 December 2017); doi: 10.1117/12.2292741

SPIE. Event: Photonics Prague 2017, 2017, Prague, Czech Republic 


\title{
Efficient conceptual design for LED-based pixel light vehicle headlamps \\ Marcel Philipp Held ${ }^{* a}$, Roland Lachmayer ${ }^{\mathrm{a}}$ \\ ${ }^{a}$ Institute of Product Development, Leibniz Universität Hannover, Welfengarten 1A, 30167 Hannover
}

\begin{abstract}
High-resolution vehicle headlamps represent a future-oriented technology that can be used to increase traffic safety and driving comfort. As a further development to the current Matrix Beam headlamps, LED-based pixel light systems enable ideal lighting functions (e.g. projection of navigation information onto the road) to be activated in any given driving scenario. Moreover, compared to other light-modulating elements such as DMDs and LCDs, instantaneous LED on-off toggling provides a decisive advantage in efficiency.

To generate highly individualized light distributions for automotive applications, a number of approaches using an LED array may be pursued. One approach is to vary the LED density in the array so as to output the desired light distribution. Another notable approach makes use of an equidistant arrangement of the individual LEDs together with distortion optics to formulate the desired light distribution. The optical system adjusts the light distribution in a manner that improves resolution and increases luminous intensity of the desired area.

An efficient setup for pixel generation calls for one lens per LED. Taking into consideration the limited space requirements of the system, this implies that the luminous flux, efficiency and resolution image parameters are primarily controlled by the lens dimensions.

In this paper a concept for an equidistant LED array arrangement utilizing distortion optics is presented. The paper is divided into two parts. The first part discusses the influence of lens geometry on the system efficiency whereas the second part investigates the correlation between resolution and luminous flux based on the lens dimensions.
\end{abstract}

Keywords: Automotive Lighting, LED-based Pixel light system, High-resolution headlamps, Étendue, Imaging optics

\section{INTRODUCTION}

The current resolution of Matrix-LED headlamps is limited to 84 pixels. Improving resolution will make it possible to eliminate glaring caused by other road users or by retroreflective road signs [1] and to project navigation and driving information onto the road. Image generation can be realized using spatial light modulators (SLM) [2][3] such as digital micromirror devices (DMDs) [4], liquid crystal displays (LCDs) or LED arrays [5].

LCDs and DMDs are subtractive image generators which generate the desired light distribution by the absorption of specified pixels from a resolution consisting of up to several million pixels. LED arrays, on the other hand, generate the light distribution by addressing each LED separately. Because of limited installation space LEDs currently have a resolution of only a few thousand pixels [6].

Current SLM-based headlamp concepts make use of a low-resolution light source to illuminate the SLM as a whole [7]. The system efficiency is thus lowered due to absorption of the stray pixels. Compared to this, an LED array has the advantage that the light distribution can be manipulated by simply switching off unused LEDs and increasing the system efficiency as a result. In order to realize the full potential of such an advantage, the efficiency of the imaging optics has to be maximized. Therefore, the optics have to simultaneously capture the maximized luminous flux from each semiconductor light source and focus the LED image uniformly on an intermediate image plane. Both of these tasks have to be accomplished while ensuring a good contrast ratio of the image. This is accomplished by limiting the acceptance angle of the distorting optics.

*held@ipeg.uni-hannover.de; phone +49 511762 3720; fax +49 511762 4506; ipeg.uni-hannover.de

Photonics, Devices, and Systems VII, edited by Karel Fliegel, Petr Páta,

Proc. of SPIE Vol. 10603, 1060315 · C 2017 SPIE · CCC code:

0277-786X/17/\$18 - doi: $10.1117 / 12.2292741$ 


\section{DESIGN CONFIGURATION OF A HIGH-RESOLUTION VEHICLE HEADLAMP}

An individual light distribution can be realized either via the adjustment of the light source or the distortion optics. To enhance safety and comfort, it is important that these distributions have a high resolution. Ultimately, the lighting unit of a high-resolution headlamp is reduced to a simplified model as shown in Figure 1 below. It consists of a light source, its corresponding power supply and control unit as well as primary optics (PO). The PO capture the emitted light at a specific acceptance angle and project the light on to an intermediate image plane. The SLM can be placed on this plane as an option as well. The light is then guided via secondary optics (SO) onto the road. Each element is electrically, thermally and optically (except the power supply and control unit) subject to irreversible energy losses.

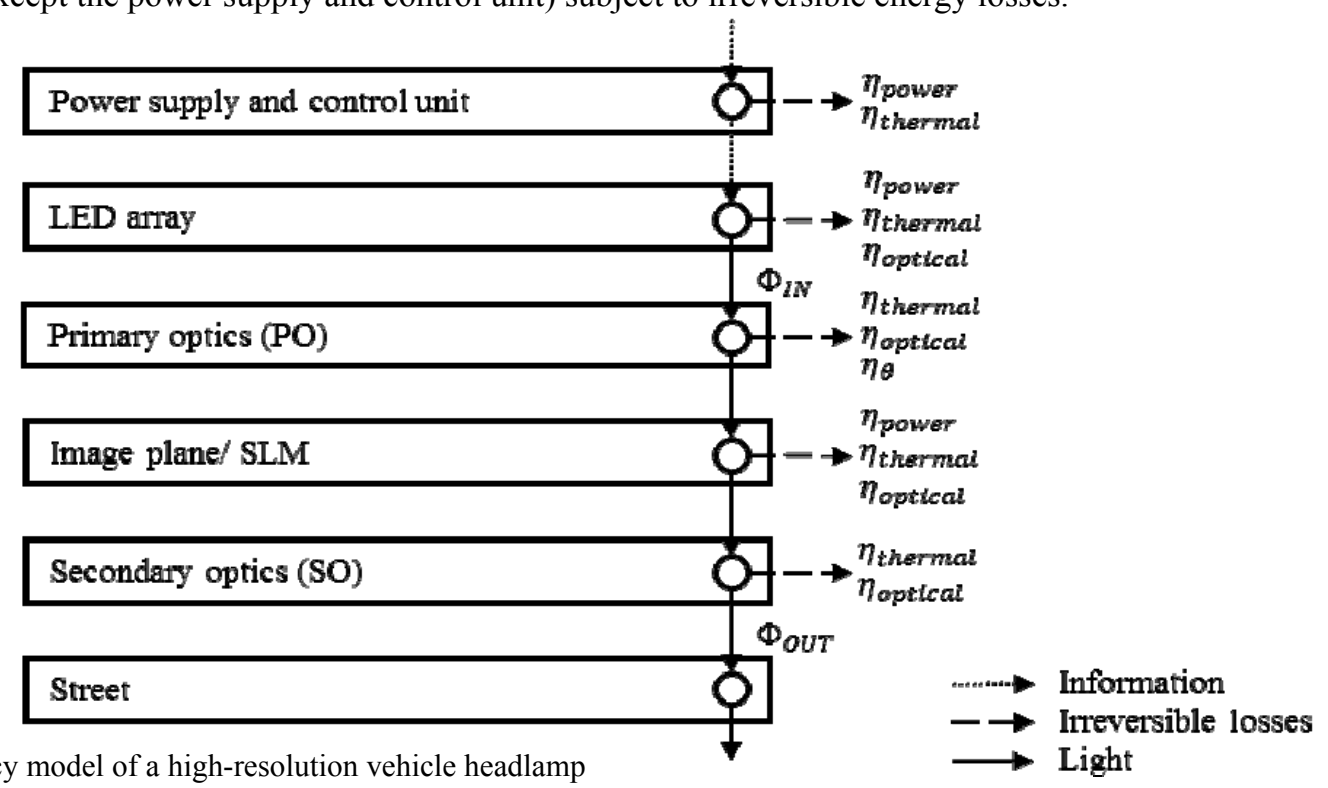

Figure 1. System efficiency model of a high-resolution vehicle headlamp

Equation 1 shows the correlation between the luminous flux of the LED array and the efficiencies of the respective optical elements. The highest impact on improving the system efficiency can be achieved by capturing as much emitted light from the semiconductor light source with the $\mathrm{PO}$ as possible i.e. enhancing the primary optics geometric efficiency $\eta_{\theta}$ [8]. Other efficiencies are mostly default property values. $\eta_{\theta}$ is highly influenced by the LED arrangement, the distance $r$ between the LED and the lens and the semi lens edge length $d$ related to Lambertian emitter characteristics:

$$
\Phi_{I N}=\frac{\Phi_{\text {OUT }}}{\eta_{\theta} \cdot \eta_{P O} \cdot \eta_{S L M} \cdot \eta_{S O} \cdot \eta_{\text {thermal }} \cdot \eta_{\text {power }}} .
$$

To form a distinct image having reasonable intensity and a gapless resolution, the output of each LED has to be acquired by the optical system. Therefore, at least one lens per LED (one pixel) becomes necessary (Figure 2).

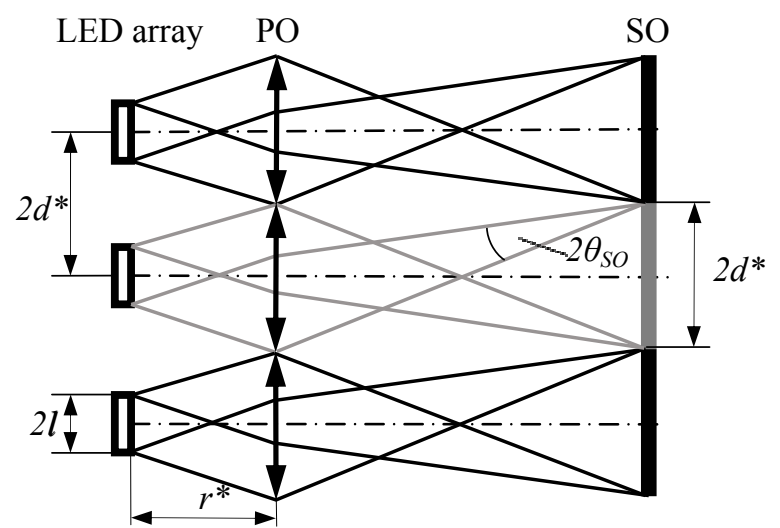

Figure 2. LED array with imaging optics projecting a gapless light distribution 
For a gapless, non-overlapping imaging system, a minimum lens edge length is directly proportional to the pixel diameter and is limited by the LED specific pixel pitch. In the simplest case of a square lens geometry the total emitting area of the LED array is defined by the lens edge length $2 d$ and the resolution $n$ (see equation 2).

$$
A=n \cdot 4 d^{2}
$$

\section{CONCEPTUAL DESIGNS}

For imaging optics where each LED has its own PO, the pixel pitch edge length equals the maximum lens edge length $2 d$. This results in a resolution that is limited on the intermediate image plane. Depending on the LED arrangement, various geometries are suitable but may not utilize the whole pixel pitch for the guidance of light. Kusch et al. demonstrates the suitability of different lens geometries to a set of LED arrangements [9]. In case of a Cartesian LED arrangement it is assumed that a square lens geometry uses the maximum area to guide the light and overcome the dark gaps produced by the pixel pitch [10].

There are basically two approaches to create a high-resolution light distribution. One approach is to locally increase the LED density (Figure 3A). The disadvantage of an increased LED density is an increased thermal stress at higher densities. A locally dense LED array leads to a lower semi lens edge length and a resulting drop in efficiency. This is due to the fact that an LED is a Lambertian emitter. The LED density is also limited by the minimum pixel pitch and therefore the resolution may not be improved significantly. Owing to the aforementioned reasons, this approach is not pursued further.

A second approach is to use an equidistant LED arrangement with a constant LED density (Figure 3B). In this case, the efficiency is globally identical and the LEDs suffer the same thermal stress (except the ones on the sides). However, the secondary optical system still has to be designed to generate a hot spot in the desired area [11]. To maintain a good functionality of the SO, the defined acceptance angle must not be exceeded. To summarize, it is necessary for the PO to transfer the LED-die light distribution onto the intermediate image plane at a specific acceptance angle (Figure 2). Therefore, an LED array acceptance angle that provides the optimum resolution $n$ and efficiency $\eta$ needs to be calculated.

LED Array

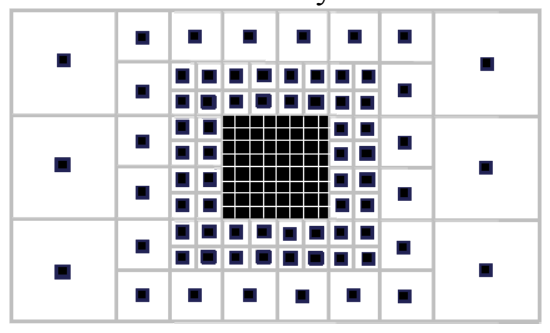

LED Array

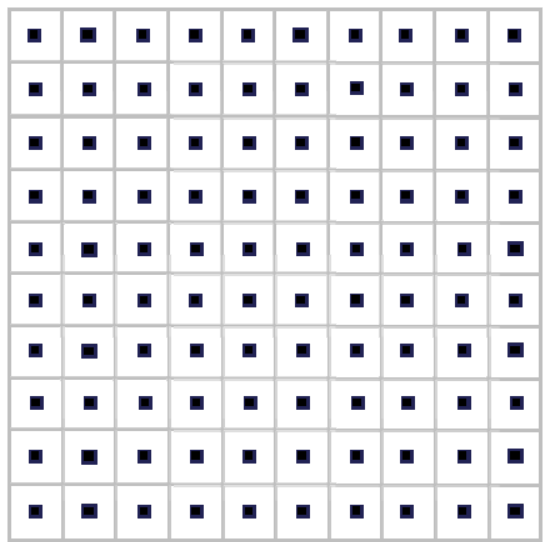

light distribution

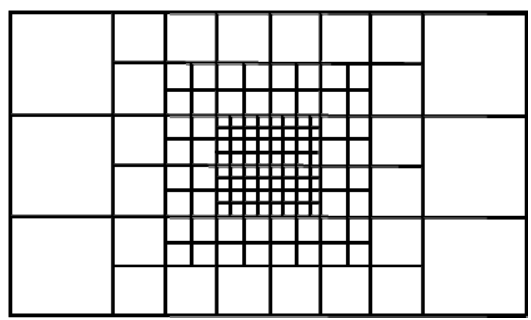

light distribution

distorting optics

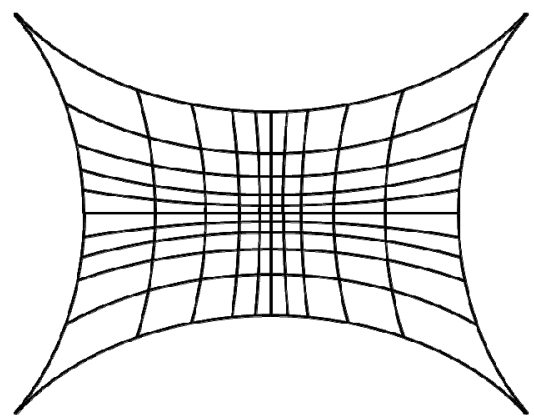

Figure 3. Conceptual designs for creating a high-resolution hot spot light distribution 


\section{INFLUENCES ON THE PRIMARY OPTICS GEOMETRIC EFFICIENCY}

To achieve an efficient projection of the light emitting area on to the intermediate image plane, a suitable position for the measurement of the PO acceptance angle has to be defined. In Figure 4 three possible measurement positions are illustrated.

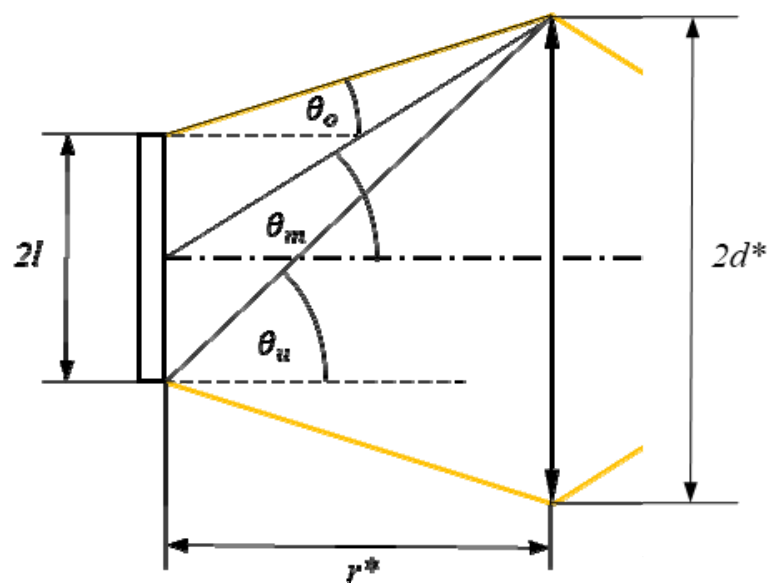

Figure 4. Three different measuring positions of the acceptance angle $\theta$

Since the maximum semi lens edge length $d$ is influenced by the acceptance angle, it is important to measure it as precisely as possible i.e. even the slightest deviation will have a high impact on the resolution of the image. Therefore, each definition needs to be standardized in accordance with the dimension $2 l$ of the light source. A simple derivation results in the following three equations:

$$
\theta_{o}=\arctan \frac{d^{*}-1}{r^{*}} \quad \text { (3a), } \quad \theta_{m}=\arctan \frac{d^{*}}{r^{*}} \quad(3 \mathrm{~b}), \quad \theta_{u}=\arctan \frac{d^{*}+1}{r^{*}}
$$

To evaluate the measuring positions, a simulation was implemented using the parameters shown in Table 1 . The light source was set to a Lambertian emitter with an emitting angle of $\pm 90^{\circ}$. Following this, the maximum angle impinging onto the detector plane (used as a substitute for the lens) was measured for a set of different $r$ values and compared to the equation results of (3a) - (3c).

Table 1. Simulation set-up parameter

\begin{tabular}{|l|r|}
\hline Parameter & Value \\
\hline light emitting area $2 l$ & $1 \mathrm{~mm}$ \\
\hline $\begin{array}{l}\text { semi edge length of the } \\
\text { detector plane }\end{array}$ & $2 \mathrm{~mm}$ \\
\hline LED emitting angle & $\pm 90 \mathrm{deg}$ \\
\hline
\end{tabular}

Table 2 shows a high deviation of equations (3a) and (3b) to the results of the simulation except for the values of equations (3c). Since this angle is a suitable approximation for the simulations, it will be used to calculate the primary optics geometric efficiency (equation 4).

Table 2. Values of the acceptance angle compared to the simulated acceptance angle for $d=2 l$

\begin{tabular}{|c|c|c|c|c|}
\hline$r$ in $\mathrm{mm}$ & $\theta_{o}$ in deg & $\theta_{m}$ in deg & $\theta_{u}$ in deg & $\theta_{\text {sim }}$ in deg \\
\hline 0,5 & 71.56 & 75.96 & 78.69 & 78.59 \\
\hline 1 & 56.31 & 63.43 & 68.19 & 67.24 \\
\hline 2 & 36.87 & 45.00 & 51.34 & 50.97 \\
\hline 3 & 26.56 & 33.69 & 39.81 & 39.66 \\
\hline 4 & 20.56 & 26.56 & 32.00 & 31.86 \\
\hline 5 & 16.69 & 21.80 & 26.56 & 26.12 \\
\hline
\end{tabular}


Based on the equation (3c), Figure 6 shows the primary optics geometric efficiency as a function of the normalized distance $r^{*}$ and normalized semi lens edge length $d^{*}$ :

$$
\eta=\frac{1}{2}(1-\cos (2 \theta))
$$

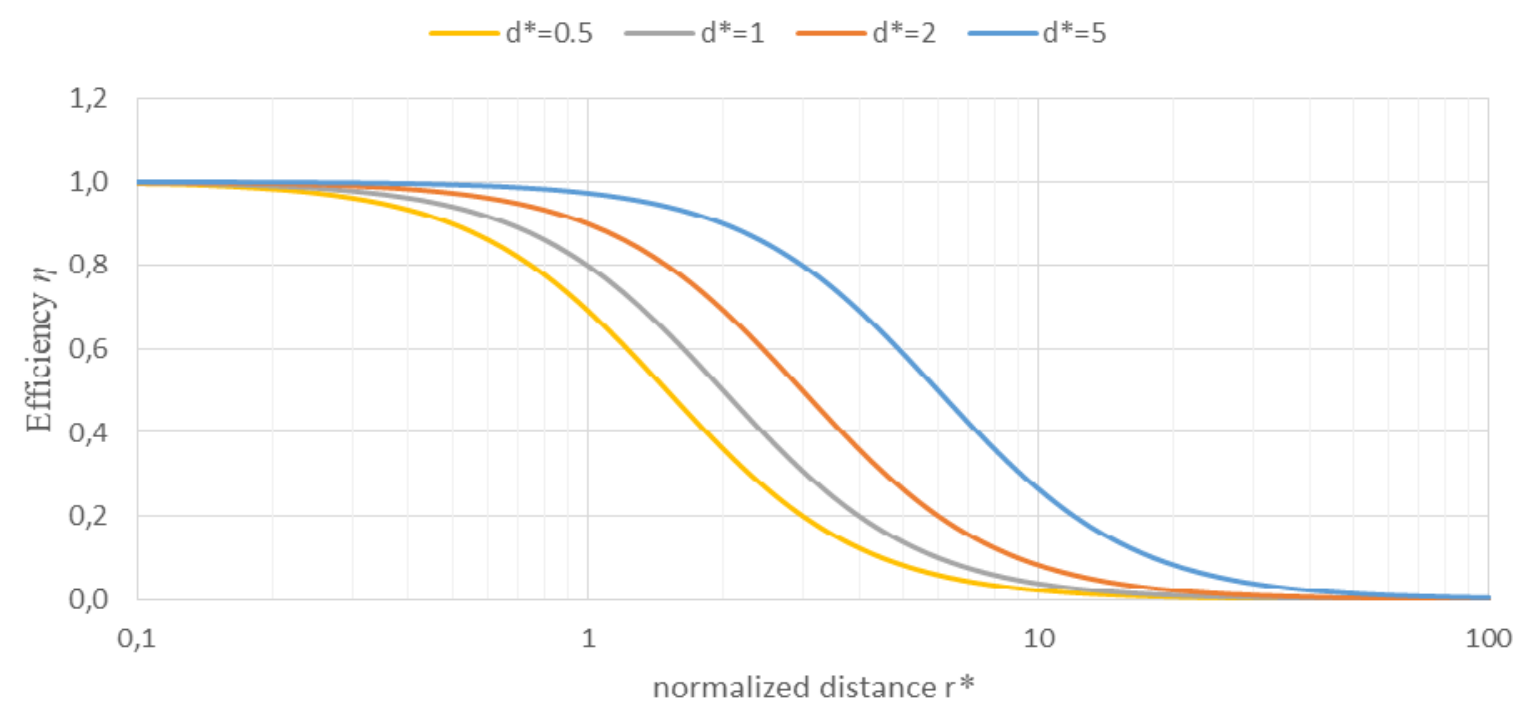

Figure 5. System efficiency based on normalized parameters $r^{*}$ and $d^{*}$

With a constant semi lens edge length $d^{*}$ an increase of the distance $r^{*}$ will always lower the efficiency. In the context of system resolution this implies that an increase of the lens edge length reduces the resolution and increases the primary optics geometric efficiency. A value less than $d^{*}=0,5$ is not reasonable for the normalized semi lens edge length since this would suggest a lens dimension smaller than the LED itself. The normalized lens edge length $d^{*}$ can be calculated by altering equation 2 to:

$$
d^{*}=\frac{1}{2 l} \sqrt{\frac{A}{n}}
$$

To design a system with a maximum luminous flux, the efficiency as well as the maximum resolution has to be considered. This means that a careful consideration of the étendue is also required. The reason for this is that a higher étendue results in a correspondingly higher luminous flux (assuming a constant luminance) [8].

The étendue $G$ is a geometric parameter which is defined as the product of the light emitting surface area $A$, the number of light sources $N$ and the corresponding acceptance angle $\theta$ of the light source:

$$
G=N \cdot A \cdot \pi \cdot \sin ^{2} \theta \text {. }
$$

As can be seen from equation 6 , the étendue is an invariant quantity. This means that it cannot be reduced without accepting irreversible energy losses. For the given case, accepting irreversible energy losses results in a reduction of the light emitting angle $\theta$. As a result, the étendue of the PO should ideally be equal to the étendue of the SO:

$$
G_{P O} \leq G_{S O} \text {. }
$$

If $G_{P O}$ is greater than $G_{S O}$, the $\mathrm{SO}$ are not able to use the higher étendue- resulting in higher losses. Thus, the smallest étendue defines the étendue of the total system. Since the étendue of the SO $G_{S O}$ is predefined by its aperture area and acceptance angle, it can be used to calculate the necessary maximum étendue of the PO. For this, the light source quantity i.e. resolution can be determined by the acceptance angle.

For the following étendue consideration the $\mathrm{SO}$ will be assumed to have an aperture area of $A=2000 \mathrm{~mm}^{2}$ and an acceptance angle of $\theta= \pm 12^{\circ}$. The étendue of the $\mathrm{SO}$ is $G_{S O}=274.6 \mathrm{~mm}^{2} \mathrm{sr}$ and expresses the recommended maximum étendue of the PO (equation 7). 


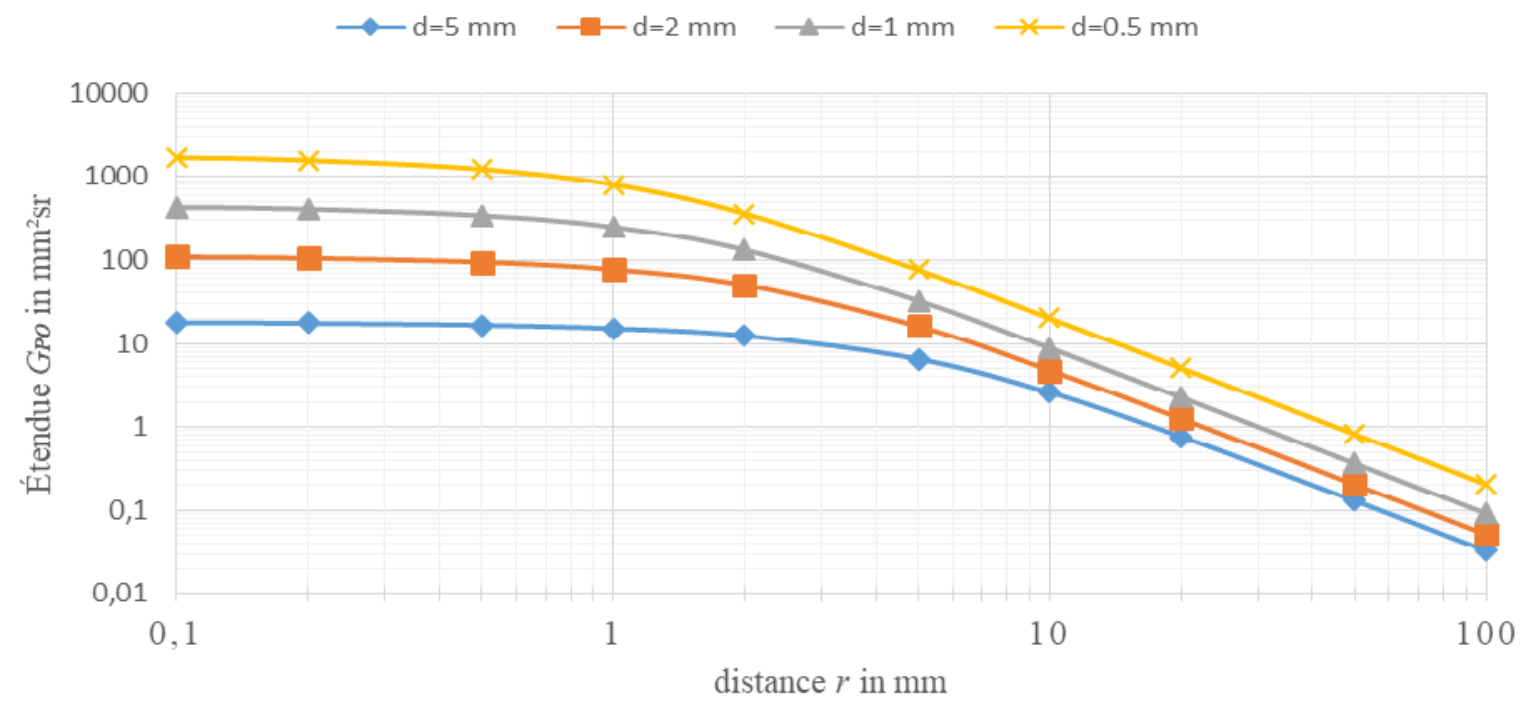

Figure 6. Étendue $G_{P O}$ as a function of distance $r$ and semi lens edge length $d$

For the LEDs we assume a light emitting area of $A=1 \mathrm{~mm}^{2}$ each, as well as a maximum LED array area equal to the SO aperture area. Thus, Figure 6 shows the étendue of the PO as a function of the distance $r$ for various semi lens edge length $d$ and resolutions $n$ respectively. To realize a maximum system resolution, a semi lens edge length of $d=0,5 \mathrm{~mm}$ is chosen (lens edge length equals the light emitting area). At a distance of approximately $r=5 \mathrm{~mm}$ a resolution of $N=2000$ pixels is achieved (equation 5). The expected efficiency is $\eta=8 \%$. A higher efficiency can be achieved by lowering the distance to $r=2 \mathrm{~mm}$ as well as increasing the semi lens edge length to $d=2 \mathrm{~mm}$. In this case the efficiency equals $\eta=69 \%$ but results in a reduced resolution of $N=125$ pixels.

For a conclusive comparison, Figure 7 shows the overall luminous flux $\Phi$ for various distances $r$ over the resolution $n$. Increasing the distance $r$ leads to a lower gradient of the luminous flux $\Phi$. For the given SO, the most suitable solution is a resolution of $N=125$ pixels at a distance of $r=2 \mathrm{~mm}$ and a resulting efficiency of $\eta=69 \%$.

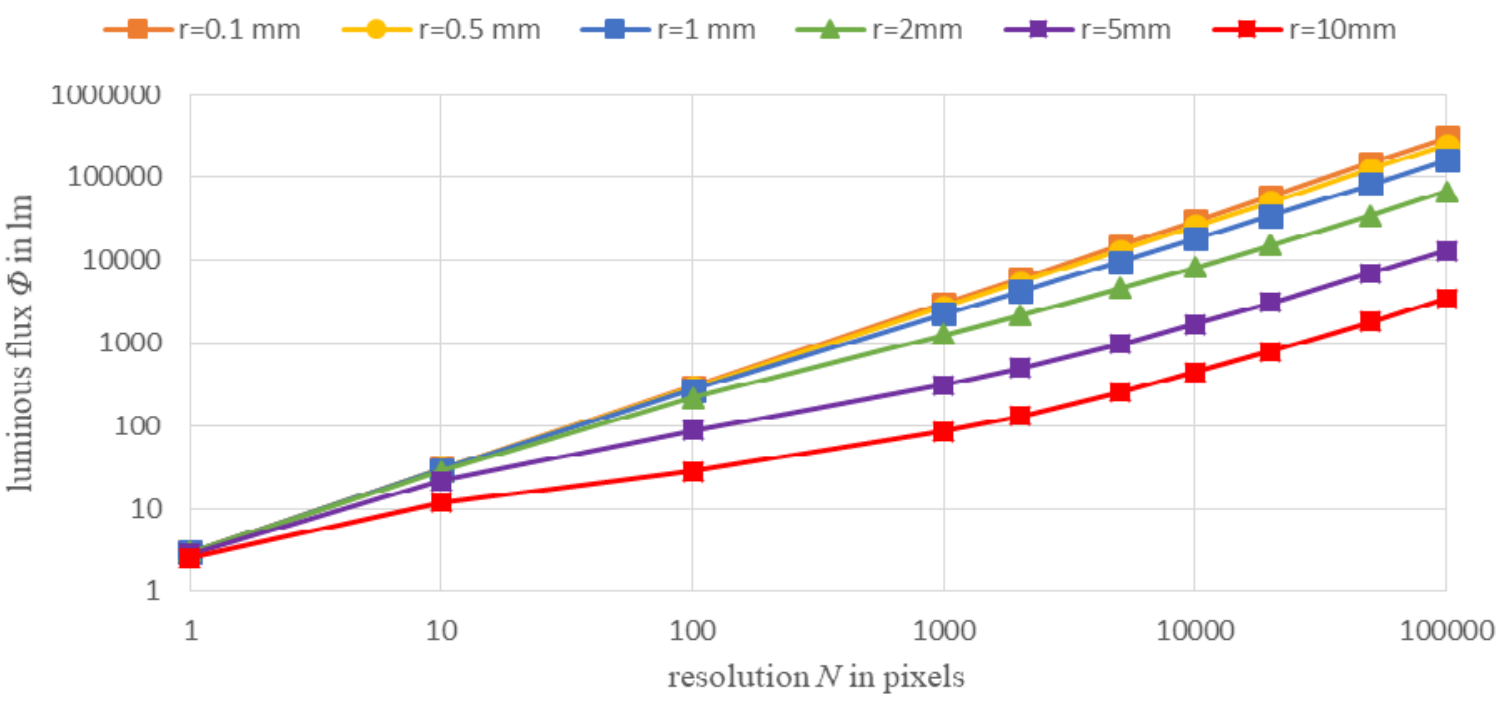

Figure 7. Overall luminous flux $\Phi$ as a function of resolution $N$ for various distances $r$ for a luminous flux of $\Phi=3 \mathrm{~lm}$ per LED 


\section{CONCLUSIONS AND OUTLOOK}

An SLM-based headlamp offers a high-resolution but lacks in system efficiency. An LED array can compensate for such a disadvantage by simply switching off unused LEDs and thus improving the system efficiency. In order to realize the full potential of such an advantage, the efficiency of the imaging LED optics has to be maximized. The primary optics geometric efficiency is mainly a function of the semi lens edge length, the distance between the light source and the PO and the given acceptance angle of the SO. First, a suitable approximation for the light emitting acceptance angle was compared to simulation results. With this approximation, the system efficiency was calculated as well as the étendue of the PO. The étendue was later used to define the maximum suitable resolution for a given SO. A high resolution can be achieved at approximately the same étendue (or luminous flux) with a lower efficiency that is used to obtain a low resolution given a higher system efficiency.

The next steps involve the implementation of the results into the PO simulation and the comparison of the simulated luminous flux with the presented values.

\section{ACKNOWLEDGEMENT}

This project was funded by the Ministry for Science and Culture of Lower Saxony within the framework of the project Tailored Light.

\section{REFERENCES}

[1] C. Jürgens, "Der kontrastadaptive Scheinwerfer," TEWISS Verlag, ISBN: 978-3-90900-015-4 (2015).

[2] X.-L. Wang, J. Ding, W.-J. Ni, C.-S. Guo, H.-T. Wang, "Generation of arbitrary vector beams with a spatial light modulator and a common path interferometric arrangement," Opt. Lett. 32, 3549-3551 (2007).

[3] E. Uzi, "Spatial Light Modulator Technology," Marcel Dekker Inc., Series: Optical engineering, ISBN: 0-82479108-8 (1995).

[4] J. Roslak, "Entwicklung eines aktiven Scheinwerfersystems zur blendungsfreien Ausleuchtung des Verkehrsraums," PhD thesis, urn:nbn:de:hbz:466-20050101459, 2005.

[5] G. Kloppenburg, A. Wolf, R. Lachmayer, "High-resolution vehicle headlamps: technologies and scanning prototype," Advanced Optical Technologies 5, Nr. 2, 147-155 (2016).

[6] T. Liebetrau, W. Pohlmann, J. Moisel, R. Kürschner, A. Pfeuffer, "Ein neuer Ansatz für hochauflösende LEDPixel-Scheinwerfer," ELIV 2015, 265-275 (2015).

[7] M. S. Dassanayake, P. Mulvanny, S. Alles, "Headlamp system with selectable beam pattern," US Patent US6497503 B1 (2002).

[8] J. Jiao, B. Wang, "High Efficiency Reflector Optics for LED Automotive Forward Lighting," Proc. SPIE 6670, Nonimaging Optics and Efficient Illumination Systems IV, 66700M (September 18, 2007); doi:10.1117/12.730524, (2007).

[9] A. Kusch, M. Wurz, M. P. Held, A. Wolf, R. Lachmayer, "LED packaging with optimized heat dissipation for a micro LED array," Smart Systems Integration, 399-402, ISBN: 987-3-95735-057-2 (2017).

[10] J. Moisel, R. Ackermann, M. Griesinger, "Adaptive Headlights utilizing LED-Arrays," Proceedings of the $8^{\text {th }}$ International Symposium on Automotive Lighting, 287-296 (2009).

[11] A. Wolf, G. Kloppenburg, R. Danov, R. Lachmayer, "DMD Based Automotive Lighting Unit," DGaO Proceedings 2016, ISSN: 1614-8436 (2016). 